\title{
Interference of Light in a Michelson-Morley Interferometer: A Quantum Optical Approach
}

\author{
Ø. Langangen, ${ }^{1,2}$ A. Vaskinn, ${ }^{3}$ and B.-S. Skagerstam ${ }^{3,4}$ \\ ${ }^{1}$ Institute of Theoretical Astrophysics, University of Oslo, P.O. Box 1029, Blindern, 0315 Oslo, Norway \\ ${ }^{2}$ Department of Biology, Centre for Ecological and Evolutionary Synthesis (CEES), University of Oslo, P.O. Box 1066, Blindern, \\ 0316 Oslo, Norway \\ ${ }^{3}$ Department of Physics, The Norwegian University of Science and Technology, 7491 Trondheim, Norway \\ ${ }^{4}$ Centre for Advanced Study (CAS), Drammensveien 78, 0271 Oslo, Norway
}

Correspondence should be addressed to B.-S. Skagerstam, bo-sture.skagerstam@ntnu.no

Received 2 May 2012; Accepted 14 July 2012

Academic Editor: Chenggen Quan

Copyright $\odot 2012 \varnothing$. Langangen et al. This is an open access article distributed under the Creative Commons Attribution License, which permits unrestricted use, distribution, and reproduction in any medium, provided the original work is properly cited.

\begin{abstract}
The temporal coherence interference properties of light as revealed by single detector intensity measurements in a MichelsonMorley interferometer (MMI) is often described in terms of classical optics. We show, in a pedagogical manner, how such features of light also can be understood in terms of a more general quantum-optics framework. If a thermal reference source is used in the MMI local oscillator port in combination with a thermal source in the signal port, the interference pattern revealed by single detector intensity measurements shows a distinctive dependence on the differences in the temperature of the two sources. A related method has actually been used to perform high-precision measurements of the cosmic microwave background radiation. The general quantum-optics framework allows us to consider any initial quantum state. As an example, we consider the interference of single photons as a tool to determine the peak angular-frequency of a single-photon pulse interfering with a single-photon reference pulse. A similar consideration for laser pulses, in terms of coherent states, leads to a different response in the detector. The MMI experimental setup is therefore an example of an optical device where one, in terms of intensity measurements, can exhibit the difference between classical and quantum-mechanical light.
\end{abstract}

\section{Introduction}

In 2006, Smoot and Mather shared the Nobel Prize in physics "for their discovery of the black-body form and anisotropy of the cosmic microwave background radiation (CMB)" [1]. These exciting discoveries were a breakthrough in modern cosmology by the CMB anisotropy and the strong validation of the black-body spectrum as predicted by the Big Bang theory. The discovery of the black-body form of the CMB spectrum and the high-precision measurement of the CMB temperature (see e.g., [2]) relied heavily on the so-called FarInfrared Absolute Spectrophotometer (FIRAS) [3] on board the Cosmic Background Explorer (COBE) [4, 5]. In short, the FIRAS is a Michelson-Morley interferometer enabling a comparison of the interference patterns between an observed source and a reference black-body source on board the COBE satellite.
In this paper, we will make use of Glauber's theory for photon detection $[6,7]$ (for a guide to the early literature see e.g., [8-10] and for textbook accounts see e.g., [11-14]) together with elementary quantum mechanics to show how the principles of the FIRAS can be understood in a straightforward manner using a quantum-optics frame-work. Interference phenomena in classical optics are described in terms of classical electro-magnetic fields. Such classical fields can be obtained in terms of expectation values of the observable electromagnetic field operators using very special quantum states, that is, coherent states (see e.g., [8-10]). Quantum states with a fixed number of photons, like single-photon states, have, however, no average electromagnetic field, and the interference effects of such states therefore requires an extension of classical considerations. The use of quantum optics enables us to actually consider arbitrary quantum 
states of the source and reference source, thereby extending the realm of classical optics.

In Section 2, we recapitulate the principles of Glaubers photon detection theory $[6,7]$ and the transformation properties of a quantum field in a beamsplitter (see e.g., [1517]). The Glauber theory of optical coherence is by now well established and plays a central role in fundamental studies of quantum interference effects of photon quantum states (see e.g., [18-20]). In Section 3, we consider temporal interference effects in the Michelson-Morley interferometer for pure quantum states like single-photon states as well as classical states corresponding to coherent states (see e.g., [810]). In Section 4, we explain the principles of interference of thermal light in the Michelson-Morley interferometer by using only vacuum as the reference source and reproduce known expressions. Here, we also show that the detection intensity obtained with a vacuum reference source does not depend on the details of signal quantum state considered, thermal or not, but only on the average number of photons present. In Section 5, we consider the full system with an observed thermal source combined with a thermal reference source. With the results obtained, we then recover the basic principle of FIRAS and how it may be used as a highprecision thermometer. In Section 6, we, finally, give some concluding remarks.

Our presentation extends a recent presentation by Donges [21] and illustrates, for example, that a quantummechanical treatment directly leads to the concept of a thermal coherence length without explicitly making use of results from classical physics like the Wiener-Khintchine theorem as in [21] and that any quantum state can be considered.

\section{The Michelson-Morley Interferometer}

We consider the classical Michelson-Morley interferometer (MMI) as illustrated in Figure 1, where the so-called temporal coherence properties of the radiation field are probed (for an early account see e.g., [22]). Figure 1 is a simplified and schematic version of the FIRAS experimental setup. In order to understand the appearance of interference effects in the MMI, we first discuss the separate parts of the MMI before we consider the full setup with the presence of a reference beam.

2.1. Glauber's Theory of Photon Detection. Let us outline a simple, but not unrealistic, model of a photon detector situated at the space-time point $(\vec{x}, t)$. In this simplified model of a photon detection process $[6,7]$, the detection of a photon is described by an annihilation of a photon at the detector which modifies the initial state $\mid$ in $\rangle$ as follows:

$$
\left.\mid \text { in }\rangle \rightarrow \vec{E}^{(+)}(\vec{x}, t) \mid \text { in }\right\rangle .
$$

Here the time-evolution is implemented in the Heisenberg picture instead of a Schrödinger picture with space-and timedependent "wave-functions" for photons. The Heisenberg picture seems actually to be mandatory since positiondependent wave functions appear to be ill defined for

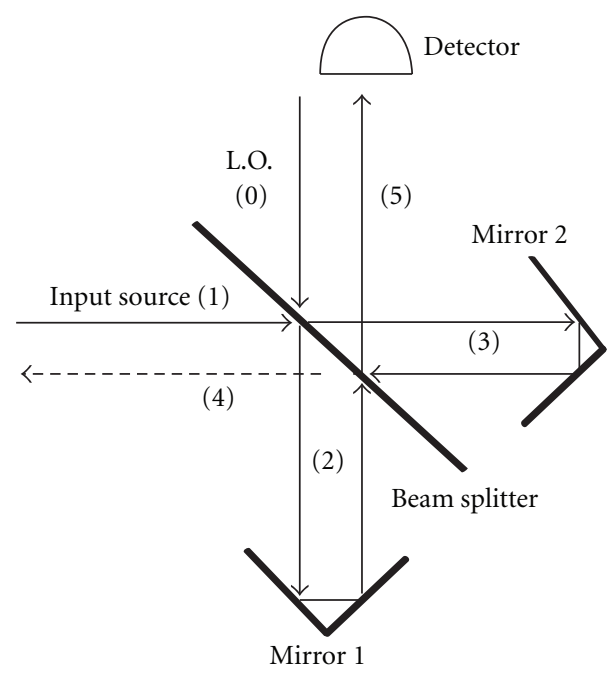

FIGURE 1: Schematic drawing of the MMI setup considered in this paper. The thick and diagonal line is representing a beam splitter with a transmission coefficient $T$. The movable mirrors 1 and 2 are identical. The numbers in the parenthesis are referring to the indices used to label the mode operators of the various light beams as discussed in the main text. The signal port (1) and the local oscillator port LO (0) are prepared with various quantum states, like Fock states, coherent states, or states with random phases like thermal states. The output mode (4) will not be considered in the present paper.

massless particles with a nonzero spin (see e.g., [23]). The observable electric field operator in Heisenberg picture, $\vec{E}(\vec{x}, t)=\sum_{m} \vec{E}_{m}(\vec{x}, t)$, is then described in terms of a suitable normal mode expansion, indexed by mode the number $m$, as a superposition of positive and negative angular-frequency contributions as follows:

$$
\vec{E}_{m}(\vec{x}, t)=\vec{E}_{m}^{(+)}(\vec{x}, t)+\vec{E}_{m}^{(-)}(\vec{x}, t),
$$

where $\vec{E}_{m}^{(+)}(\vec{x}, t)\left(\vec{E}_{m}^{(-)}(\vec{x}, t)\right)$ contains an annihilation (creation) operator for a photon with mode number $m$. According to the basic Born rule in quantum mechanics, the probability to detect the system in a final state $\mid$ out $\rangle$, after the single-photon absorption process, is then proportional to $\mid\left.\left\langle\right.$ out $\left|\vec{E}^{(+)}(\vec{x}, t)\right|$ in $\rangle\right|^{2}$. Since the exact details of the final states are, in general, unknown we sum over all possible final states $\mid$ out $\rangle$, that is, we consider $\sum_{\text {out }} \mid\left.\left\langle\right.$ out $\left|\vec{E}^{(+)}(\vec{x}, t)\right|$ in $\rangle\right|^{2}=$ $\left\langle\right.$ in $\left|\vec{E}^{(-)}(\vec{x}, t) \vec{E}^{(+)}(\vec{x}, t)\right|$ in $\rangle$, where use has been made of the completeness of all possible final states, that is, $\sum_{\text {out }} \mid$ out $\rangle$ $\langle$ out $|=1$. In general, we also have to consider not only a pure initial quantum state but also a quantum state as described by a density matrix. This leads to a description of the observed intensity $I$ which we write in the form

$$
I=\operatorname{Tr}\left[\rho_{\text {in }} \vec{E}^{(-)}(\vec{x}, t) \vec{E}^{(+)}(\vec{x}, t)\right],
$$

where $\rho_{\text {in }}$ is the density matrix describing the initial state. Since we will not be interested in the absolute normalization of the observed intensity $I$, we can neglect normalization 
constants that may enter into $I$. It is a remarkable fact that an analysis of single-photon interference in a Young interferometer using such a quantum-mechanical description of the photon detection process [24], as well as a proper experimental investigation of single-photon interference [25, $26]$, is fairly recent in the history physics.

For reasons of clarity, we will now consider a typical plane wave normal mode expansion of the electromagnetic field observable $\vec{E}(\vec{x}, t)$ in terms of a mode sum over wave-vectors $\vec{k}$ and polarization-vector labels $\lambda$, that is, we have for its positive angular-frequency part that

$$
\vec{E}^{(+)}(\vec{x}, t)=i \sum_{\vec{k} \lambda} \sqrt{\frac{\hbar \omega_{k}}{2 V \varepsilon_{0}}} \vec{\varepsilon}_{\vec{k} \lambda} a_{\vec{k} \lambda} e^{i \vec{k} \cdot \vec{x}-i \omega_{k}\left(t-t_{0}\right)},
$$

with $\omega_{k}=c|\vec{k}|$. The annihilation operator $a_{\vec{k} \lambda}$, and the corresponding unit polarization vector $\overrightarrow{\varepsilon_{k \lambda}}$, denotes the normal mode considered. $t_{0}$ is a suitable initial-time parameter which will be used, in a manner to be clarified below, to express the dependence on various propagation times for the optical paths as given in the MMI setup, a procedure as is well known in classical optics (see e.g., [12, 22]). If the direction of the light beams considered is well defined, the dependence of the detector position $\vec{x}$ can be neglected in the expression for $I$. In general, spatial modulations of the measured intensity are expected $[27,28]$. A theoretical analysis of such effects along lines as discussed in the literature (see e.g., $[29,30]$ ) will, however, not enlighten the issues we are addressing in the present paper. $V$ is a quantization volume that will be allowed to be arbitrarily large at an appropriate late stage of our calculations.

Since the dependence of normalization constants will be irrelevant, and since we will only consider polarizationindependent optical devices, we make use of a scalar notation. We therefore suppress the wave vector and polarization labels and with $\omega \equiv \omega_{k}$ we write $a(\omega) \equiv a_{\vec{k} \lambda}$ such that $\left[a(\omega), a^{\dagger}(\omega)\right]=\delta_{\omega \omega^{\prime}}$ in terms of a discrete Kronecker delta $\delta_{\omega \omega^{\prime}}$. We also make use of the following convenient notation [12] for the positive angular-frequency part of the electric field at the position of the detector at time $t$ :

$$
E^{(+)}(t)=i \sum_{\omega} \sqrt{\delta \omega} \omega^{1 / 2} a(\omega) e^{i \phi(t)}
$$

with the prescription $\sum_{\omega} \delta \omega \rightarrow \int d \omega \omega^{d-1}$ in the infinite volume limit $V \rightarrow \infty$ and where $d$ is the number of spacedimensions. Here $\phi(t) \equiv-\omega(t-\tau)$ now denotes a modedependent optical phase which explicitly takes the sourcedetection propagation time into account in terms of the time-delay $\tau$, which will be evaluated in detail for the MMIsetup below. In (5), the mode operator $a(\omega)\left(\equiv a_{5}(\omega)\right.$ in Figure 1) will later be expressed in terms of a superposition of the input mode operators $\left(\equiv a_{0}(\omega)\right.$ and $\equiv a_{1}(\omega)$ in Figure 1$)$. If the detector time $t$ enters explicitly into the detection intensity equation (3), we will perform a time average which corresponds to a finite detector time-resolution window.
The corresponding time average of the observed intensity $I \equiv I(t)$ will be denoted by $\langle I\rangle$, that is,

$$
\langle I\rangle=\frac{1}{T_{\mathrm{int}}} \int_{-T_{\mathrm{int}} / 2}^{T_{\mathrm{int}} / 2} d t I(t),
$$

where the time $T_{\text {int }}$ of integration, as for example, the time during which an actual measurement proceeds, is chosen to be sufficiently large in comparison with typical time scales of intensity fluctuations. The time-averaged observed intensity $\langle I\rangle$ will in general, as we will see explicitly below, be a function of a time-delay $\tau$ depending on the actual experimental set up.

For a finite quantization volume $V, \omega$ can be regarded to be discrete and it may be assumes that $d=1$. When appropriate we will, however, also consider $d=3$ in order to compare with related results in the literature $[21,22]$. Our main results will, however, not be very sensitive to the choice of $d$. Using the same notation as above, a single-photon quantum state $|f\rangle$, with an angular-frequency distribution given by $f \equiv f(\omega)$, is then given by

$$
|f\rangle=\sum_{\omega} \sqrt{\delta \omega} f(\omega)\left|1_{\omega}\right\rangle \equiv\left(f, a^{\dagger}\right)|0\rangle,
$$

where $\left|1_{\omega}\right\rangle=a^{\dagger}(\omega)|0\rangle$ denotes a single-photon state with angular-frequency $\omega$, normalized according to $\left\langle 1_{\omega} \mid 1_{\omega^{\prime}}\right\rangle=$ $\delta_{\omega \omega^{\prime}}$, and where $|0\rangle$ denotes the vacuum state. We also make use of the notation $\left(f, a^{\dagger}\right)=\left(f^{*}, a\right)^{\dagger} \equiv \sum_{\omega} \sqrt{\delta \omega} f(\omega) a^{\dagger}(\omega)$. The state $|f\rangle$ above is an eigenstate of the number operator $\hat{N}=\sum_{\omega} a^{\dagger}(\omega) a(\omega)$, that is, a Fock state, with, of course, an eigenvalue corresponding to one particle present. Normalization of the state $|f\rangle$ for $d=1$ therefore corresponds to

$$
\langle f \mid f\rangle=1=\sum_{\omega} \delta \omega f^{*}(\omega) f(\omega)=\int_{0}^{\infty} d \omega|f(\omega)|^{2},
$$

in the large-volume $V$ limit. In order to make our presentation quantitative, we will, for reasons of simplicity, consider real-valued, single-photon, angular-frequency distributions $f(\omega)$ such that

$$
f(\omega)=\frac{1}{N} \exp \left(-\frac{(\omega-\bar{\omega})^{2}}{2 \sigma^{2}}\right),
$$

with a mean angular-frequency $\bar{\omega}$ and width $\sigma$ and where the normalization constant $N$ is given by

$$
|N|^{2}=\frac{\sigma \sqrt{\pi}}{2}\left(1+\frac{2}{\sqrt{\pi}} \int_{0}^{\bar{\omega} / \sigma} d x e^{-x^{2}}\right) .
$$

This choice of frequency distribution makes it possible to actually carry out all relevant expressions analytically. In obtaining the properly normalized expression Equation (9), we keep $\omega \geq 0$. It may, however, sometimes be possible to extend the range of angular frequencies to arbitrarily negative values in (8), so that $|N|^{2}=\sigma \sqrt{\pi}$, with an exponential small error, which makes some of the expressions more tractable and transparent. With our choice of beam parameters below, it turns out that such an 
approximation plays only a minor role with regard to our numerical evaluations where we make use of $\omega \geq 0$. It is now clear that $\langle f|\vec{E}(\vec{x}, t)| f\rangle \equiv 0$, that is, a single-photon state has no average electromagnetic field.

There are, however, quantum states with a nonzero expectation value of the electromagnetic field operator $\vec{E}(\vec{x}, t)$ are like coherent states. Conventional coherent states $|f\rangle_{c}$, as expressed in terms of the single-photon distribution $f$, can for example, be obtained using a multimode displacement operator (see e.g., [8-10]), that is,

$$
|f\rangle_{c}=e^{\left(f, a^{\dagger}\right)-\left(f^{*}, a\right)}|0\rangle=e^{-\langle f \mid f\rangle / 2} e^{\left(f, a^{\dagger}\right)}|0\rangle
$$

such that

$$
a(\omega)|f\rangle_{c}=(\delta \omega)^{1 / 2} f(\omega)|f\rangle_{c}
$$

and hence

$$
{ }_{c}\langle f|\hat{N}| f\rangle_{c}=\sum_{\omega} \delta \omega|f(\omega)|^{2}
$$

We also observe that the expectation value of the singlecomponent electromagnetic field operator $E^{(+)}(t)$ as defined in (5) is given by

$$
{ }_{c}\left\langle f\left|E^{(+)}(t)(\vec{x}, t)\right| f\right\rangle_{c}=i \sum_{\omega} \delta \omega \omega^{1 / 2} f(\omega) e^{i \phi(t)},
$$

which therefore corresponds to a classical electromagnetic field. Classical interference effects as expressed in terms of such expectation values, which are nonlinearly dependent of the quantum states considered, are, of course, not directly linked to quantum-mechanical interference effects which are linearly dependent of quantum states. The use of quantum optics clarifies the connection between these views on interference effects.

The input quantum states to be considered in Figure 1, will, of course, correspond to the replacements $a(\omega) \rightarrow$ $a_{0}(\omega)$ and $a(\omega) \rightarrow a_{1}(\omega)$, respectively in the definition of the states considered above.

2.2. Transformation in the Beam Splitter. Next, we consider a dispersionless beam splitter with frequency-independent transmittance $T$ and reflectance $R$. If we assume a prefect beam splitter, where all light is either reflected or transmitted, we have $R+T=1$. The input annihilation operators $a_{0}(\omega)$ and $a_{1}(\omega)$ of the beam splitter will then transform according to (see e.g., [15-17])

$$
\begin{aligned}
& a_{2}(\omega)=\sqrt{T} a_{0}(\omega)+i \sqrt{R} a_{1}(\omega), \\
& a_{3}(\omega)=\sqrt{T} a_{1}(\omega)+i \sqrt{R} a_{0}(\omega),
\end{aligned}
$$

where $a_{0}(\omega)$ and $a_{1}(\omega)$ are the LO and signal port mode annihilation operators, and $a_{2}(\omega), a_{3}(\omega)$ the output annihilation mode operators corresponding to the transmitted and reflected modes, respectively. The transformation rules for the corresponding creation operators follows from (15) by Hermitian conjugation. The two independent sets of output annihilation and creation operators will then obey the required canonical commutation relations $\left[a_{2}(\omega), a_{2}^{\dagger}(\omega)\right]=$ $\left[a_{3}(\omega), a_{3}^{\dagger}(\omega)\right]=1$. The phase shift between the transmitted and reflected part, described by the complex numbers in (15) will play an important role below as is also the case in the famous Hong-Ou-Mandel two-photon correlation experiment [31] and related investigations (see e.g., [3235]).

A light beam arriving at the beam splitter after being reflected in the mirrors 1 and 2 will, in general, be phase shifted, that is, expressed in terms of mode operators this process corresponds to the propagation time replacements in (5):

$$
\begin{aligned}
& a_{2}(\omega) e^{i \phi(t)} \longrightarrow a_{2}(\omega) e^{i \phi_{2}(t)}, \\
& a_{3}(\omega) e^{i \phi(t)} \longrightarrow a_{3}(\omega) e^{i \phi_{3}(t)},
\end{aligned}
$$

due to difference in optical path lengths with $\phi_{2}=-\omega(t-$ $\left.\tau_{2}\right), \phi_{3}=-\omega\left(t-\tau_{3}\right)$ in terms of time-delays $\tau_{2}$ and $\tau_{3}$. The reflections at the identical mirrors in the MMI setup (Figure 1) will also introduce phase-shifts, but they are equal for the two light beams and can therefore be neglected all together. A light beam passing through the beam splitter after reflection at the mirrors will, again, transform according to (15) and we therefore, finally, obtain an expression for the mode operator describing incident light on the photon detector in (5), that is,

$$
\begin{aligned}
a_{5}(\omega) e^{i \phi(t)} \longrightarrow & \sqrt{T} a_{2}(\omega) e^{i \phi_{2}(t)}+i \sqrt{R} a_{3}(\omega) e^{i \phi_{3}(t)} \\
= & a_{0}(\omega)\left(T e^{i \phi_{2}(t)}-R e^{i \phi_{3}(t)}\right) \\
& +a_{1}(\omega)\left(i \sqrt{T R} e^{i \phi_{2}(t)}+i \sqrt{T R} e^{i \phi_{3}(t)}\right) .
\end{aligned}
$$

For reasons of clarity, we will consider a 50/50 beam splitter, which also makes interference effects dependent only on the phases present, that is, we make the choice $T=R=1 / 2$. The corresponding electromagnetic field operator to be used in Glauber's theory of photon detection, is therefore given by

$$
\begin{aligned}
E^{(+)}(t)=i \sum_{\omega} \frac{1}{2} \sqrt{\delta \omega} \omega^{1 / 2} \\
\times\left(a_{0}(\omega)\left(e^{i \phi_{2}(t)}-e^{i \phi_{3}(t)}\right)\right. \\
\left.+i a_{1}(\omega)\left(e^{i \phi_{2}(t)}+e^{i \phi_{3}(t)}\right)\right) .
\end{aligned}
$$

\section{Interference of Fock States and Coherent States}

Let us now specifically consider single photons in the signal and local ports, that is, the following initial Fock state as follows:

$$
\mid \text { in }\rangle=\left|f_{s}\right\rangle \otimes\left|f_{l o}\right\rangle
$$

with single-photon angular-frequency distributions according to (9). In the numerical results to be presented, we will use the same spectral widths $\sigma$ but with $\bar{\omega} \rightarrow \bar{\omega}_{s}=3 \sigma$ for 
the signal port and $\bar{\omega} \rightarrow \bar{\omega}_{l o}=3.15 \sigma$ or $\bar{\omega}_{l o}=2.85 \sigma$ for the local oscillator port. We now observe that the action of the electromagnetic field operator $E^{(+)}(t)$, as given by (18), on this initial state is given by

$$
\begin{aligned}
\left.E^{(+)}(t) \mid \text { in }\right\rangle= & i \sum_{\omega} \frac{1}{2} \delta \omega \omega^{1 / 2} \\
\times & \left(i f_{s}(\omega) e^{i \phi_{2}(t)}\left(1+e^{i \omega \tau}\right)|0\rangle \otimes\left|f_{l o}\right\rangle\right. \\
& \left.\quad+f_{l o}(\omega) e^{i \phi_{2}(t)}\left(1-e^{i \omega \tau}\right)\left|f_{s}\right\rangle \otimes|0\rangle\right),
\end{aligned}
$$

where we have made use of the fact that $\phi_{3}(t)-\phi_{2}(t)=$ $\omega\left(\tau_{3}-\tau_{2}\right) \equiv \omega \tau$, which now defines the optical time-delay $\tau$. The nonnormalized state $E^{(+)}(t) \mid$ in $\rangle$ describes an entangled combination of the two orthogonal state vectors $|0\rangle \otimes\left|f_{l o}\right\rangle$ and $\left|f_{s}\right\rangle \otimes|0\rangle$ corresponding to the absorption of one photon from the signal source or from the local oscillator, respectively. Apart from an overall normalization constant, each expansion coefficient, that is, the corresponding probability amplitude, contains information about the single-photon interference for the signal or the local oscillator photon, respectively. As we have discussed above, the probability for the detection of one photon will, according to Glauber's theory of photon detection, be proportional to the matrix element $\left\langle\right.$ in $\left|E^{(-)}(t) E^{(+)}(t)\right|$ in $\rangle$, that is, the absolute modulus square $\left|E^{(+)}(t)\right|$ in $\rangle\left.\right|^{2}$ of the nonnormalized state $E^{(+)}(t) \mid$ in $\rangle$. A time average over the time $t$ according to (6) with $T_{\text {int }} \gg$ $1 / \sigma$ leads to a Dirac $\delta\left(\omega-\omega^{\prime}\right)$-function and therefore makes any double-sum over frequencies into a single-sum. In the large-volume limit and for $d=1$, we then obtain

$$
\begin{aligned}
\langle I\rangle(\tau)= & \frac{1}{2 T_{\text {int }}} \int_{0}^{\infty} d \omega 2 \pi \\
& \quad \times\left(\omega\left|f_{s}(\omega)\right|^{2}(1+\cos \omega \tau)\right. \\
& \left.\quad+\omega\left|f_{l o}(\omega)\right|^{2}(1-\cos \omega \tau)\right) .
\end{aligned}
$$

The single-photon detection intensity $\langle I\rangle(\tau)$ is then a sum of two single-photon interference patterns. We notice the appearance of a relative minus sign in the local oscillator photon contribution, which has its origin in the relative phase-shift between the transmission-reflection and transmission-transmission coefficients of a beamsplitter alluded to in connection with (15) and (17). If $\bar{\omega}_{s}=\bar{\omega}_{l o}$ then the detector cannot distinguish between a photon from the signal or the local source with the same spectral widths $\sigma$ and the two interference effects will exactly cancel against each other with the result that $\langle I\rangle(\tau)$ will be independent of $\tau$.

If $\bar{\omega}_{s}, \bar{\omega}_{l o} \gg \sigma$, then, within a good numerical approximation, we can first replace the linear $\omega$ dependence in (21) with $\bar{\omega}_{s}$ and $\bar{\omega}_{l o}$ in front of the corresponding angular-frequency distributions, and then extend the integration to include

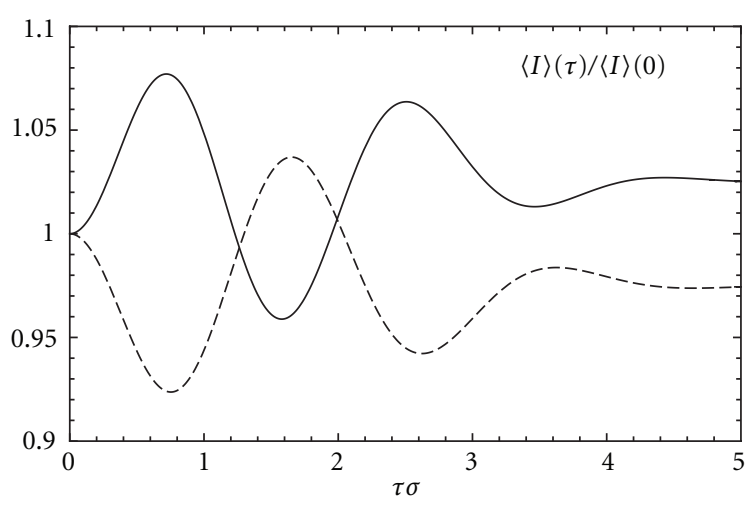

FIGURE 2: The normalized single-photon intensity $\langle I\rangle(\tau) /\langle I\rangle(0)$ as a function of the dimensionless time-delay $\tau \sigma$ for the case of single-photon states in the signal and the LO ports with the same spectral width $\sigma$ but with different mean frequencies. Two different examples are plotted with $\bar{\omega}_{s}=3 \sigma: \bar{\omega}_{l o}=3.15 \sigma$ with (solid line) or $\bar{\omega}_{l o}=2.85 \sigma$ (dashed line). The asymptotic values of $\langle I\rangle(\tau) /\langle I\rangle(0)$ can be obtained from the expression (22) in the main text, that is, $\left(1+\bar{\omega}_{l o} / \bar{\omega}_{s}\right) / 2$.

arbitrarily negative angular-frequencies. One then finds that $\langle I\rangle(0) \simeq 2 \pi \bar{\omega}_{s} / T_{\text {int }}$ and

$$
\begin{aligned}
\frac{\langle I\rangle(\tau)}{\langle I\rangle(0)} \simeq \frac{1}{2}(1 & +\frac{\bar{\omega}_{l o}}{\bar{\omega}_{s}} \\
& \left.+e^{-\sigma^{2} \tau^{2} / 4}\left(\cos \tau \bar{\omega}_{s}-\frac{\bar{\omega}_{l o}}{\bar{\omega}_{s}} \cos \tau \bar{\omega}_{l o}\right)\right),
\end{aligned}
$$

where the interference effects are exponentially sensitive to the spectral width $\sigma$ of the single-photon angulardistributions in a fashion similar to the spectral width dependence in the famous Hong-Ou-Mandel two-photon experiment [31]. The asymptotic value of $\langle I\rangle(\tau) /\langle I\rangle(0)$ is given by $\left(1+\bar{\omega}_{l o} / \bar{\omega}_{s}\right) / 2$. In Figure 2 , we exhibit $\langle I\rangle(\tau) /\langle I\rangle(0)$ according to (21) with the choice as in (19). With a given reference distribution $\left|f_{l o}(\omega)\right|^{2}$ of the local oscillator one can, for example, now in principle infer the common spectral width $\sigma$ of the single-photon sources as well as the corresponding angular-frequency $\bar{\omega}_{s}$. A related experimental situation is actually discussed in [36] for a general singlephoton state, that is, not necessarily a pure quantum state.

In the case of coherent states in the signal and local ports, with single-particle state angular-frequency distributions $f_{s}(\omega)$ and $f_{l o}(\omega)$ as above, (20) is now modified according to

$$
\begin{aligned}
\left.E^{(+)}(t) \mid \text { in }\right\rangle= & i \sum_{\omega} \frac{1}{2} \delta \omega \omega^{1 / 2} \\
& \times\left(i f_{s}(\omega) e^{i \phi_{2}(t)}\left(1+e^{i \omega \tau}\right)\right. \\
& \left.\quad+f_{l o}(\omega) e^{i \phi_{2}(t)}\left(1-e^{i \omega \tau}\right)\right)\left|f_{s}\right\rangle_{c} \otimes\left|f_{l o}\right\rangle_{c}
\end{aligned}
$$

which is not an entangled state. This is so since the removal of a photon from a coherent state does not change the state since it actually contains infinitely many photons, that is, 


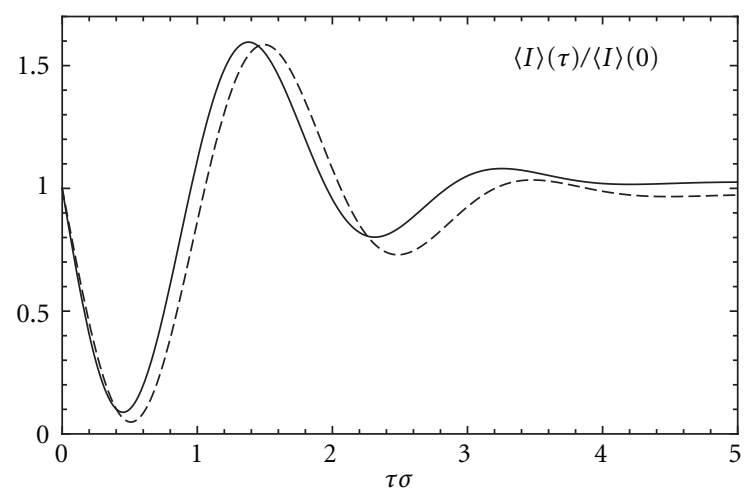

FIgURE 3: The normalized intensity $\langle I\rangle(\tau) /\langle I\rangle(0)$ as a function of the time-delay $\tau \sigma$ for the case of coherent states as generated by the same single-photon states as in Figure 2. Two different examples are plotted with $\bar{\omega}_{s}=3 \sigma: \bar{\omega}_{l o}=3.15 \sigma$ with (solid line) or $\bar{\omega}_{l o}=$ $2.85 \sigma$ (dashed line). The asymptotic values of $\langle I\rangle(\tau) /\langle I\rangle(0)$ are as in Figure 2.

$a(\omega)|f\rangle_{c}=(\delta \omega)^{1 / 2} f(\omega)|f\rangle_{c}$ according to (12). The intensity $\langle I\rangle(\tau)$ according to $(21)$ is, for real-valued single-photon distributions $f_{s}(\omega)$ and $f_{l o}(\omega)$, therefore replaced by

$$
\begin{aligned}
\langle I\rangle(\tau)=\frac{1}{2 T_{\text {int }}} \int_{0}^{\infty} d \omega \omega 2 \pi( & \left|f_{s}(\omega)\right|^{2}(1+\cos \omega \tau) \\
& +\left|f_{l o}(\omega)\right|^{2}(1-\cos \omega \tau) \\
& \left.-2 f_{s}(\omega) f_{l o}(\omega) \sin \omega \tau\right),
\end{aligned}
$$

which now contains an additional interference contribution as compared with (21). In Figure 3, we exhibit $\langle I\rangle(\tau) /\langle I\rangle(0)$ with coherent states generated by the same choice of singlephoton states as in Figure 2. The interference pattern is, as in Figure 2, sensitive to the actual angular-frequency distributions. With a given reference distribution $\left|f_{l o}(\omega)\right|^{2}$ of the local oscillator, one can now infer the common spectral width $\sigma$ of the coherent state sources as well as the corresponding angular-frequency $\bar{\omega}_{s}$. By a comparison with Figure 2, we conclude that the MMI setup is sensitive to the actual form of the initial quantum states despite the fact that we are only considering single-photon detection processes. With the same approximations leading to (22), we find an additional contribution to $\langle I\rangle(\tau) /\langle I\rangle(0)$, that is,

$$
\begin{array}{r}
\frac{\langle I\rangle(\tau)}{\langle I\rangle(0)} \simeq \frac{1}{2}\left(\left(1+\frac{\bar{\omega}_{l o}}{\bar{\omega}_{s}}\right)\left(1-e^{-\delta \bar{\omega}^{2} / 4 \sigma^{2}-\sigma^{2} \tau^{2} / 4} \sin \bar{\omega} \tau\right)\right. \\
\left.+e^{-\sigma^{2} \tau^{2} / 4}\left(\cos \tau \bar{\omega}_{s}-\frac{\bar{\omega}_{l o}}{\bar{\omega}_{s}} \cos \tau \bar{\omega}_{l o}\right)\right),
\end{array}
$$

with $\delta \bar{\omega} \equiv\left(\bar{\omega}_{s}-\bar{\omega}_{l o}\right)$ and $\bar{\omega} \equiv\left(\bar{\omega}_{l o}+\bar{\omega}_{s}\right) / 2$. In particular, and more important, we notice that when $\bar{\omega} \equiv \bar{\omega}_{l o}=\bar{\omega}_{s}$ we can write

$$
\frac{\langle I\rangle(\tau)}{\langle I\rangle(0)} \simeq 1-e^{-\sigma^{2} \tau^{2} / 4} \sin \tau \bar{\omega},
$$

which now explicitly depends on $\tau$ in contrast to the initial Fock state considerations above in which case $\langle I\rangle(\tau) \simeq$ $\langle I\rangle(0)$.

\section{Interference of Thermal Light in the Michelson-Morley Interferometer}

4.1. Thermal Light. For the readers convenience, let us first outline a simple and quantum mechanical description of thermal black body radiation at an absolute temperature $T$. Black body radiation is, basically, light with random phases. For a single mode with angular-frequency $\omega$ and if $|n\rangle=$ $a^{\dagger}(\omega)^{n}|0\rangle / \sqrt{n !}$ denotes a $n$-photon state, the density matrix describing the thermal light is given by

$$
\rho(\omega)=\sum_{n=0}^{\infty} p_{n}(\omega)\left|n_{\omega}\right\rangle\left\langle n_{\omega}\right|,
$$

in terms of the Bose-Einstein distribution:

$$
p_{n}(\omega)=\left(\frac{\bar{n}(\omega, T)}{1+\bar{n}(\omega, T)}\right)^{n} \frac{1}{1+\bar{n}(\omega, T)},
$$

with

$$
\bar{n}(\omega, T)=\frac{1}{\exp \left(\hbar \omega / k_{B} T\right)-1} .
$$

The state $\rho(\omega)$ corresponds to an extreme value of the von Neumann entropy $S$, that is,

$$
S=-k_{B} T \operatorname{Tr}[\rho(\omega) \ln \rho(\omega)]
$$

subjected to the constraints (see e.g., $[13,22]$ ):

$$
\begin{gathered}
\operatorname{Tr}[\rho(\omega)]=1, \\
\operatorname{Tr}\left[a^{\dagger}(\omega) a(\omega) \rho(\omega)\right]=\bar{n}(\omega, T) .
\end{gathered}
$$

The random, or chaotic, nature of the quantum state $\rho(\omega)$ corresponds to a phase-independent Glauber-Sudarshan $\mathcal{P}(\alpha)$-representation (see e.g., [37-42]) in terms of a coherent state, that is,

$$
\rho(\omega)=\int d^{2} \alpha \mathcal{P}(\alpha)|\alpha\rangle\langle\alpha|,
$$

using a single-mode coherent state $|\alpha\rangle=\exp \left(\alpha a^{\dagger}(\omega)-\right.$ $\left.\alpha^{*} a(\omega)\right)|0\rangle$. For thermal light, one finds that

$$
\mathcal{P}(\alpha)=\frac{1}{\pi \bar{n}(\omega, T)} \exp \left(-\frac{|\alpha|^{2}}{\bar{n}(\omega, T)}\right),
$$

which obeys the normalization condition:

$$
\operatorname{Tr}[\rho(\omega)]=\int d^{2} \alpha \mathcal{P}(\alpha)=1
$$

as well as

$$
\operatorname{Tr}\left[a^{\dagger}(\omega) a(\omega) \rho(\omega)\right]=\int d^{2} \alpha P(\alpha)|\alpha|^{2}=\bar{n}(\omega, T) .
$$

A multimode system at thermal equilibrium is then described in terms of a tensor product $\rho(T)=\otimes_{\omega} \rho(\omega)$, where we have performed the replacement $\alpha \rightarrow \alpha(\omega)$ in (32) and (33). The Glauber-Sudarshan $\mathcal{P}(\alpha)$-representation for the state $\rho(T)$ is now, in particular, useful in our considerations since the response in a single-photon detector can be obtained immediately from the previous results for coherent light using an average procedure. 
4.2. Thermal Light in the Signal Port and Vacuum in the Local Oscillator Port. We are now in the position to consider a density matrix describing a system where we have thermal light in the signal port and vacuum in the LO port, that is, the initial density matrix $\rho_{\text {in }}$ of the total system is given by

$$
\rho_{\text {in }}=\rho(T) \otimes(|0\rangle\langle 0|)_{l o} .
$$

By making use of [25], with $f_{s}(\omega) \rightarrow \alpha(\omega)$ and $f_{l o}(\omega) \rightarrow 0$, and then performing an average over $\alpha(\omega)$ according to (35), we immediately obtain the following result:

$$
\langle I\rangle(\tau)=\frac{1}{2 T_{\text {int }}} \int_{0}^{\infty} d \omega 2 \pi \omega^{d} \bar{n}(\omega, T)(1+\cos \omega \tau),
$$

in $d$ space dimensions.

In passing, we notice the important fact that (37) is, in fact, valid for any physical quantum state of the form (36), where $\rho(T)$ is replaced with any density matrix $\rho(\omega)$ not necessarily thermal, due to the generality of the Glauber-Sudarshan $P(\alpha)$-representative equation (32). The interference effects as exhibited by the MMI setup, therefore, actually only depends on the, in general, angular-frequency dependent mean number $\operatorname{Tr}\left[a^{\dagger}(\omega) a(\omega) \rho(\omega)\right]=n(\omega)$ and not on other features of the actual quantum state $\rho(\omega)$.

By a straightforward change of the variable of integration in (37), and with $a \equiv \tau k_{B} T / \hbar$, we then find for a thermal quantum state $\rho(T)$ that

$$
\frac{\langle I\rangle(\tau)}{\langle I\rangle(0)}=\frac{1}{2}\left[1+\frac{1}{J(d)} \int_{0}^{\infty} d x\left(\frac{x^{d} \cos (a x)}{\exp (x)-1}\right)\right],
$$

where $J(d)$ can be expressed in terms of gamma and Riemann's $\zeta$ functions, that is,

$$
J(d)=\Gamma(1+d) \zeta(d+1)=\Gamma(1+d) \sum_{n=1}^{\infty} \frac{1}{n^{d+1}} .
$$

Particular values are $J(1)=\pi^{2} / 6$ and $J(3)=\pi^{4} / 15$. In the case of $d=3$, we, therefore, recover the well-known expression for $\langle I\rangle(\tau) /\langle I\rangle(0)$ [22] as also discussed in [21]. In, for example, $d=3$ it is actually possible to carry out the relevant integral in the expression equation (38) for $\langle I\rangle(\tau) /\langle I\rangle(0)$ analytically with the result

$$
\frac{\langle I\rangle(\tau)}{\langle I\rangle(0)}=\frac{1}{2}\left[1+15\left(\frac{2+\cosh (2 a \pi)}{\sinh (a \pi)^{4}}-\frac{3}{(a \pi)^{4}}\right)\right] .
$$

Equation (40) shows that interference effects have a power-law sensitivity for larger $a$. In Figure 4 , we show $\langle I\rangle(\tau) /\langle I\rangle(0)$ for varying values of $a$ in the case with $d=3$ and one infers a characteristic coherence length $l_{c}$ of thermal light in the MMI of the form:

$$
l_{c} \simeq 1.5 \frac{\hbar c}{k_{B} T},
$$

as also discussed in [21].

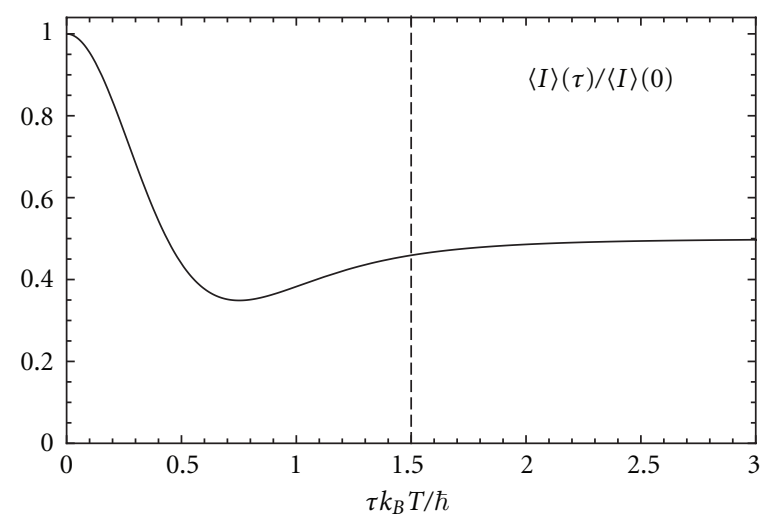

Figure 4: The normalized intensity $\langle I\rangle(\tau) /\langle I\rangle(0)$ as a function of $a \equiv \tau k_{B} T_{0} / \hbar$ for the case of thermal light in the signal port and vacuum in the LO port and for $d=3$. We infer a characteristic thermal coherence time $\tau_{c}$ in terms of $a \simeq 1.5$ (dashed vertical line), that is, $\tau_{c} \simeq 1.5 \hbar / k_{B} T$.

\section{Thermal Light in the Signal and Local Ports}

As we have seen above, with a vacuum in the LO port and with a signal thermal source the single-photon detection process exhibits an interference pattern. We now investigate what happens if we have thermal light with a temperature $T_{0}$ in the LO port and thermal light with temperature $T_{1}$ in the signal port. For this setup, the corresponding initial density matrix becomes

$$
\rho_{\text {in }}=\rho\left(T_{1}\right) \otimes \rho\left(T_{0}\right) .
$$

By making use of this density matrix as well as the same methods as described in Section 4.2 by performing independent averages over $f_{s}(\omega)$ and $f_{l_{0}}(\omega)$ in (24) according to (35), we immediately obtain, in the large-volume limit, the following result:

$$
\begin{array}{r}
\langle I\rangle(\tau)=\frac{1}{2 T_{\text {int }}} \int_{0}^{\infty} d \omega \omega^{d} 2 \pi\left(\bar{n}\left(\omega, T_{1}\right)(1+\cos \omega \tau)\right. \\
\left.+\bar{n}\left(\omega, T_{0}\right)(1-\cos \omega \tau)\right) .
\end{array}
$$

In obtaining this result, we notice that the last term in (24), suitably extended to complex-valued $f_{s}(\omega)$ and $f_{l o}(\omega)$, averages to zero due to the chaotic nature, that is, phaseindependence of thermal light according to (32) and (33). The relative intensity then takes the form $(d=3)$ as follows:

$$
\begin{aligned}
\frac{\langle I\rangle(\tau)}{\langle I\rangle(0)}= & \frac{1}{2}\left(1+\left(\frac{T_{0}}{T_{1}}\right)^{4}\right) \\
& +\frac{15}{2 \pi^{4}} \int_{0}^{\infty} d x \frac{x^{3}}{\exp (x)-1} \\
& \times\left(\cos \left(a_{1} x\right)-\left(\frac{T_{0}}{T_{1}}\right)^{4} \cos \left(a_{0} x\right)\right),
\end{aligned}
$$




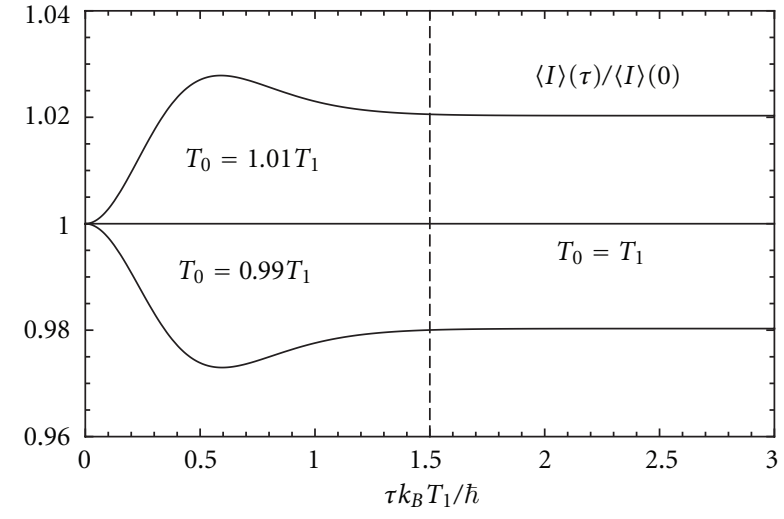

Figure 5: The same as in Figure 4 but with thermal light in both ports with $T_{1}=1.01 T_{0}$ (upper curve), $T_{1}=0.99 T_{0}$ (lower curve), and $T_{1}=T_{0}$ (solid line). The vertical dashed line corresponds to the thermal coherence length $l_{c}=c \tau_{c}$ with $\tau_{c} k_{B} T_{1} / \hbar \simeq 1.5$.

where $a_{0}=\tau k_{B} T_{0} / \hbar$ and $a_{1}=\tau k_{B} T_{1} / \hbar$. The integrals in (44) can, again, be solved analytically in a fashion similar to the integral in (38), that is,

$$
\begin{aligned}
\frac{\langle I\rangle(\tau)}{\langle I\rangle(0)}=\frac{1}{2}(1 & \left.+\left(\frac{T_{0}}{T_{1}}\right)^{4}\left(1-15 \frac{2+\cosh \left(2 a_{0} \pi\right)}{\sinh \left(a_{0} \pi\right)^{4}}\right)\right) \\
+ & \frac{15}{2}\left(\frac{2+\cosh \left(2 a_{1} \pi\right)}{\sinh \left(a_{1} \pi\right)^{4}}\right) .
\end{aligned}
$$

Due to the presence of hyperbolic functions in (45), we observe that $\langle I\rangle(\tau) /\langle I\rangle(0)$ approaches its asymptotic value $\left(1+\left(T_{0} / T_{1}\right)^{4}\right) / 2$ exponentially fast as a function of the time-delay $\tau$ in contrast to the power-law dependence in (40). It is now of interest to study the behavior of (45) when the temperatures $T_{0}$ and $T_{1}$ of the local oscillator and signal, respectively are varied. In Figure 5, we exhibit the interference when $T_{0}$ is slightly smaller or larger than $T_{1}$ as a function of the parameter $\tau k_{B} T_{1} / \hbar$ for $d=3$. The corresponding interference, of course, disappears when the two temperatures are equal. The sensitivity of the interference pattern with regard to the difference in temperatures of the source and the reference temperature, that is, of the local source, constitutes the basic ingredient of the FIRAS setup. We also observe that the coherence length $l_{c}$ for this MMI setup is roughly the same as in Section 4.2 since the two temperatures of the source considered are close to each other.

\section{Conclusions and Final Remarks}

We have seen that the interference of thermal light in the Michelson-Morley interferometer can be described, in a straightforward manner, by making use of Glauber's theory of photon detection and elementary quantum theory of the electromagnetic field. Furthermore, we have seen the emergence of a natural coherence length $l_{c} \simeq 1.5 \hbar c / k_{B} T$ of thermal light in the MMI. The result for thermal light in both the signal and the local oscillator ports shows that the interference pattern is sensitive to the difference in temperature of the two sources. This is the basic principle used by the FIRAS on board the COBE satellite in order to perform high-precision measurements of the temperature and the spectrum of the cosmic micro-wave background radiation.

It may first appear surprising that nonmonochromatic and chaotic/thermal light, with random phases, exhibits interference effects since it can be argued that a quantum state with a fixed number of photons has an undetermined "quantum phase" (see e.g., [43-48]). A mixed quantum state of such photon-number eigenstates therefore have no intrinsic and well-defined phase. As we, however, have seen the interference effects are naturally obtained in terms of the phase-dependent normal-mode expansion of the quantized electromagnetic field and its corresponding timeevolution rather than in terms of a Schrödinger picture and possible phases of quantum states (see e.g., the comments in [49]).

Since we have been considering initial quantum states in terms of a fixed number of photons as well as "classical" states, corresponding to coherent states with an infinite number of photons present, a quantum-mechanical language is mandatory. The signal and local ports of the MMI setup corresponds to independent input sources. It is, of course, a well-known experimental fact that independent photon sources can give rise to interference effects (see e.g., [5055]). Despite the fact that such interference effects are well established, the interpretation of them can, nevertheless, gives rise to interesting issues regarding the very fundamental aspects of the quantum-mechanical world (see e.g., [56]) when considering, in particular, interference effects using single-photon sources.

We have seen that for multimode systems the quantum nature of these independent sources actually affects the nature of the single-photon intensity measurements. We have already mentioned that the angular-frequency distribution of a single photon can be measured using a similar experimental setup as the MMI considered in the present paper [36]. With a vacuum state in the local oscillator port and a singlephoton signal angular-frequency distribution $f(\omega)$ of the form considered in (9), one finds, using (21), that

$$
\frac{\langle I\rangle(\tau)}{\langle I\rangle(0)} \simeq \frac{1}{2}\left(1+e^{-\sigma^{2} \tau^{2} / 4} \cos \tau \bar{\omega}_{s}\right) .
$$

We conclude by noticing that a characteristic exponential behavior as in (46) for single-photon interference has recently been observed by measuring the photoluminescence signal of a single quantum dot [57] using a MichelsonMorley interferometer (for a related study of interference effects of dissimilar photon sources, see [58]).

\section{Acknowledgments}

This research has made use of NASA's Astrophysics Data System. The research was supported by the Research Council of Norway through Grants 170935/V30 and FRINAT-191564, and by NTNU. One of the authors (B.-S. Skagerstain) wishes to thank Professor F. G. Scholtz for a generous and stimulating hospitality during a joint NITheP and Stias, Stellenbosch 
(SA), workshop in 2009, Professors M. Reid and D. V. Ahluwalia, University of Christchurch, NZ, J. R. Klauder, University of Florida, Gainesville, USA, P. S. Riseborough, Temple University, USA., the TH Division at CERN, and the Centre for Advanced Study (CAS), Oslo, for hospitality when the present work was in progress.

\section{References}

[1] Nobel Prize Website, http://www.nobelprize.org/.

[2] D. J. Fixsen and J. C. Mather, "The spectral results of the farinfrared absolute spectrophotometer instrument on COBE," Astrophysical Journal Letters, vol. 581, no. 2, pp. 817-822, 2002.

[3] J. C. Mather, D. J. Fixsen, and R. A. Shafer, "Design for the COBE far-infrared absolute spectrophotometer," in Society of Photo-Optical Instrumentation Engineers (SPIE) Conference Series, M. S. Scholl, Ed., vol. 2019 of Proceedings of SPIE, pp. 168-179, 1993.

[4] J. C. Mather, "The cosmic background explorer (COBE)," Optical Engineering, vol. 21, no. 4, pp. 769-774, 1982.

[5] J. C. Mather, Section 2.8 in Questions of Modern CosmologyGalileo's Legacy, Edited by M. D'Onofrio and C.Burigana, Springer, Berlin, Germany, 2009.

[6] R. J. Glauber, "The quantum theory of optical coherence," Physical Review, vol. 130, no. 6, pp. 2529-2539, 1963.

[7] R. J. Glauber, "Optical coherence and photon statistics," in Quantum Optics and Electronics, C. DeWitt, A. Blandin, and C. Cohen-Tannoudji, Eds., Les Houches, p. 621, Gordon and Breach, New York, NY, USA, 1965.

[8] J. R. Klauder and B.-S. Skagerstam, Coherent States-Applications in Physics and Mathematical Physics, World Scientific, Singapore, 1985, (2nd edition in Chinese, Beijing, 1986).

[9] B.-S. Skagerstam, "Coherent states-some applications in quantum field theory and particle physics," in Coherent States: Past, Present, and the Future, D. H. Feng, J. R. Klauder, and M. R. Strayer, Eds., World Scientific, Singapore, 1994.

[10] J. R. Klauder, "The current state of coherent states," in Proceedings of the the 7th ICSSUR Conference, Boston, Mass, USA, 2001.

[11] J. R. Klauder and E. C. G. Sudarshan, Fundamentals of Quantum Optics, W.A. Benjamin, New York, NY, USA, 1968, and Dover, New York, NY, USA, 2006.

[12] L. Mandel and E. Wolf, Optical Coherence and Quantum Optics, Cambridge University Press, Cambridge, UK, 1995.

[13] M. O. Scully and M. S. Zubairy, Quantum Optics, Cambridge University Press, Cambridge, UK, 1997.

[14] B. E. S. Saleh and M. C. Teich, Fundamentals of Photonics, Wiley, New York, NY, USA, 2nd edition, 2007.

[15] B. Yurke, S. L. McCall, and J. R. Klauder, "SU(2) and SU(1,1) interferometers," Physical Review A, vol. 33, no. 6, pp. 40334054, 1986.

[16] Z. Y. Ou and L. Mandel, "Derivation of reciprocity relations for a beam splitter from energy balance," American Journal of Physics, vol. 57, pp. 66-67, 1989.

[17] R. A. Campos, B. E. A. Saleh, and M. C. Teich, "Quantummechanical lossless beam splitter: $\mathrm{SU}(2)$ symmetry and photon statistics," Physical Review A, vol. 40, no. 3, pp. 1371-1384, 1989.

[18] L. Mandel, "Quantum effects in one-photon and two-photon interference," Reviews of Modern Physics, vol. 71, no. 2, pp. S274-S282, 1999.
[19] A. Zeilinger, "Experiment and the foundations of quantum physics," Reviews of Modern Physics, vol. 71, no. 2, pp. S288S297, 1999.

[20] A. Zeilinger, G. Weihs, T. Jennewein, and M. Aspelmeyer, "Happy centenary, photon," Nature, vol. 446, no. 7133, p. 342, 2007.

[21] A. Donges, "The coherence length of black-body radiation," European Journal of Physics, vol. 19, no. 3, pp. 245-249, 1998.

[22] L. Mandel and E. Wolf, "Coherence properties of optical fields," Reviews of Modern Physics, vol. 37, no. 2, pp. 231-287, 1965.

[23] B.-S. Skagerstam, "Localization of massless spinning particles and the berry phase," in On Klauders Path: A Field TripFestschrift for John R. Klauder on Occasion of His 60th Birthday, G. G. Emch, G. C. Hegerfeldt, and L. Streit, Eds., pp. 209-222, World Scientific, 1994.

[24] D. F. Walls, "A simple field theoretic description of photon interference," American Journal of Physics, vol. 45, pp. 952-956, 1977.

[25] P. Grangier, G. Roger, and A. Aspect, "Experimental evidence for a photon anticorrelation effect on a beam splitter: a new light on single-photon interference," Europhysics Letters, vol. 1, no. 4, pp. 173-179, 1986.

[26] A. Aspect and P. Grangier, "Wave-particle duality for single photons," Hyperfine Interactions, vol. 37, no. 1-4, pp. 1-17, 1987.

[27] T. J. Herzog, J. G. Rarity, H. Weinfurter, and A. Zeilinger, "Frustrated two-photon creation via interference," Physical Review Letters, vol. 72, no. 5, pp. 629-632, 1994.

[28] G. Scarcelli, A. Valencia, and Y. Shih, "Experimental study of the momentum correlation of a pseudothermal field in the photon-counting regime," Physical Review A, vol. 70, no. 5, Article ID 051802, 4 pages, 2004.

[29] L. J. Wang, X. Y. Zou, and L. Mandel, "Induced coherence without induced emission," Physical Review A, vol. 44, no. 7, pp. 4614-4622, 1991.

[30] P. W. Milonni, H. Fearn, and A. Zeilinger, "Theory of twophoton down-conversion in the presence of mirrors," Physical Review A, vol. 53, no. 6, pp. 4556-4566, 1996.

[31] C. K. Hong, Z. Y. Ou, and L. Mandel, "Measurement of subpicosecond time intervals between two photons by interference," Physical Review Letters, vol. 59, no. 18, pp. 2044-2046, 1987.

[32] Y. H. Shih and C. O. Alley, "New type of einstein-podolskyrosen-bohm experiment using pairs of light quanta produced by optical parametric down conversion," Physical Review Letters, vol. 61, no. 26, pp. 2921-2924, 1988.

[33] M. A. Steinberg, P. G. Kwiat, and R. Y. Chiao, "Measurement of the single-photon tunneling time," Physical Review Letters, vol. 71, no. 5, pp. 708-711, 1993.

[34] P. G. Kwiat, A. M. Steinberg, and R. Y. Chiao, "Observation of a quantum eraser: a revival of coherence in a two-photon interference experiment," Physical Review A, vol. 45, no. 11, pp. 7729-7739, 1992.

[35] D. V. Strekalov, T. B. Pittman, and Y. H. Shih, "What we can learn about single photons in a two-photon interference experiment," Physical Review A, vol. 57, no. 1, pp. 567-570, 1998.

[36] W. Wasilewski, P. Kolenderski, and R. Frankowski, "Spectral density matrix of a single photon measured," Physical Review Letters, vol. 99, no. 12, Article ID 123601, 4 pages, 2007.

[37] E. C. G. Sudarshan, "Equivalence of semiclassical and quantum mechanical descriptions of statistical light beams," Physical Review Letters, vol. 10, no. 7, pp. 277-279, 1963. 
[38] R. J. Glauber, "Coherent and incoherent states of the radiation field," Physical Review, vol. 131, no. 6, pp. 2766-2788, 1963.

[39] J. R. Klauder, J. McKenna, and D. G. Currie, "On "Diagonal" coherent-state representations for quantum-mechanical density matrices," Journal of Mathematical Physics, vol. 6, pp. 734$739,1965$.

[40] C. L. Metha and E. C. G. Sudarshan, "Relation between quantum and semi-classical description of optical coherence," Physical Review, vol. 138, no. 1, pp. B274-B280, 1965.

[41] J. R. Klauder, "Improved version of optical equivalence theorem," Physical Review Letters, vol. 16, no. 12, pp. 534-536, 1966.

[42] J. R. Klauder and B. S. K. Skagerstam, "Generalized phasespace representation of operators," Journal of Physics A, vol. 40, no. 9, pp. 2093-2105, 2007.

[43] L. Susskind and J. Glogower, "Quantum mechanical phase and time operator," Physics, vol. 1, pp. 49-61, 1964.

[44] S. M. Barnett and D. T. Pegg, "Phase in quantum optics," Journal of Physics A, vol. 19, pp. 3849-3862, 1986.

[45] S. M. Barnett and D. T. Pegg, "On the hermitian optical phase operator," Journal of Modern Optics, vol. 36, no. 1, pp. 7-19, 1989.

[46] S. M. Barnett and D. T. Pegg, "Phase properties of the quantized single-mode electro-magnetic field," Physical Review A, vol. 39, no. 4, pp. 1665-1675, 1989.

[47] S. M. Barnett and D. T. Pegg, "Quantum optical phase," Journal of Modern Optics, vol. 44, pp. 225-264, 1997.

[48] A. Bjørn and B.-S. Skagerstam, "On the quantum phase operator for coherent states," Physica Scripta, vol. 70, no. 1, pp. 2632, 2004.

[49] J. Xiong, D. Z. Cao, F. Huang, H.-G. Li, X.-J. Sun, and K. Wang, "Experimental observation of classical subwavelength interference with a pseudothermal light source," Physical Review Letters, vol. 94, no. 17, Article ID 173601, 2005.

[50] G. Magyar and L. Mandel, "Interference fringes produced by superposition of two independent maser light beams," Nature, vol. 198, no. 4877, pp. 255-256, 1963.

[51] R. L. Pfleegor and L. Mandel, "Further experiments on interference of independent photons beams at low light levels," JOSA, vol. 58, pp. 946-950, 1968.

[52] L. Mandel, "Photon interference and correlation effects produced by independent quantum sources," Physical Review A, vol. 28, no. 2, pp. 929-943, 1983.

[53] H. Paul, "Interference between independent photons," Reviews of Modern Physics, vol. 58, no. 1, pp. 209-231, 1986.

[54] F. Louradour, F. Reynaud, B. Colombeau, and C. Froehly, "Interference fringes between two separate lasers," American Journal of Physics, vol. 61, pp. 242-245, 1993.

[55] R. K. Kaltenbaek, B. Blauensteiner, M. Zukowski, M. AsPelmeyer, and A. Zeilinger, "Experimental interference of independent photons," Physical Review Letters, vol. 96, no. 24, Article ID 240502, 4 pages, 2006.

[56] R. J. Glauber, "Diracs's fameous dictum on the interference: one photon or two photons?" American Journal of Physics, vol. 63, no. 12, 1995.

[57] C. Kammerer, G. Cassabois, C. Voisin et al., "Interferometric correlation spectroscopy in single quantum dots," Applied Physics Letters, vol. 81, no. 15, pp. 2737-2739, 2002.

[58] A. J. Bennett, R. B. Patel, C. A. Nicoll, D. A. Ritchie, and A. J. Shields, "Interference of dissimilar photon sources," Nature Physics, vol. 5, no. 10, pp. 715-717, 2009. 

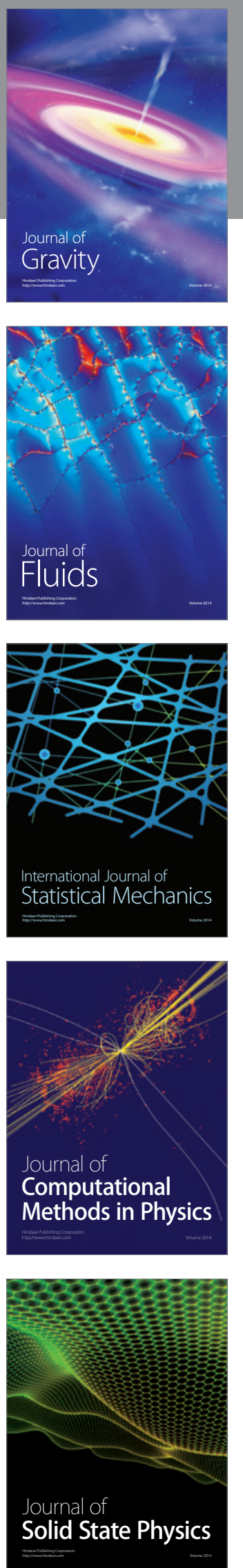

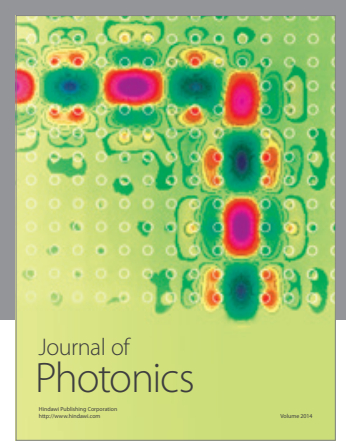

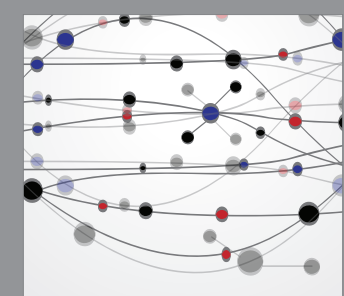

The Scientific World Journal
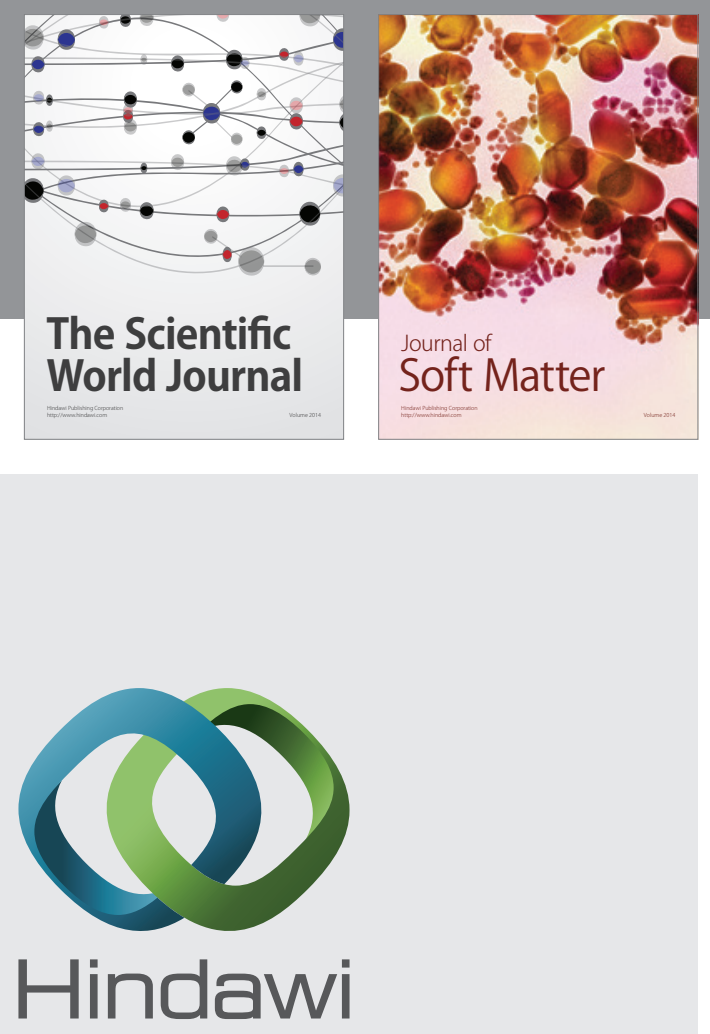

Submit your manuscripts at

http://www.hindawi.com
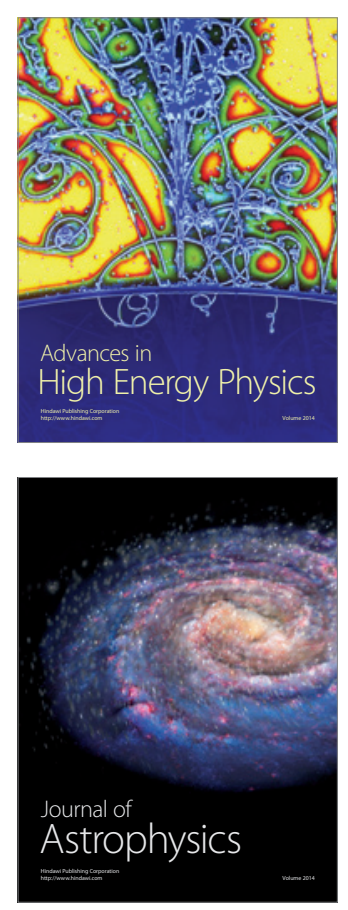
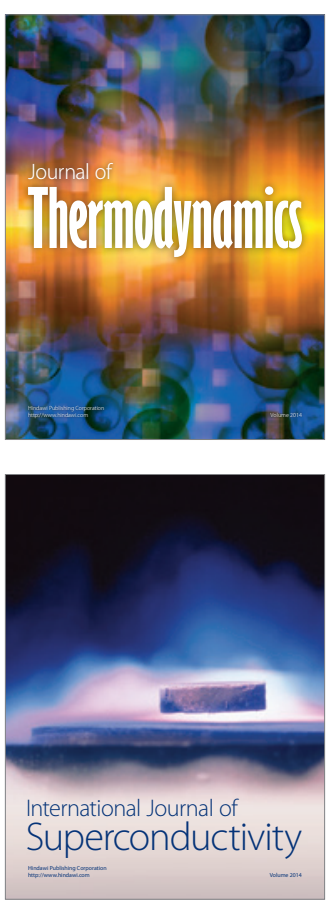
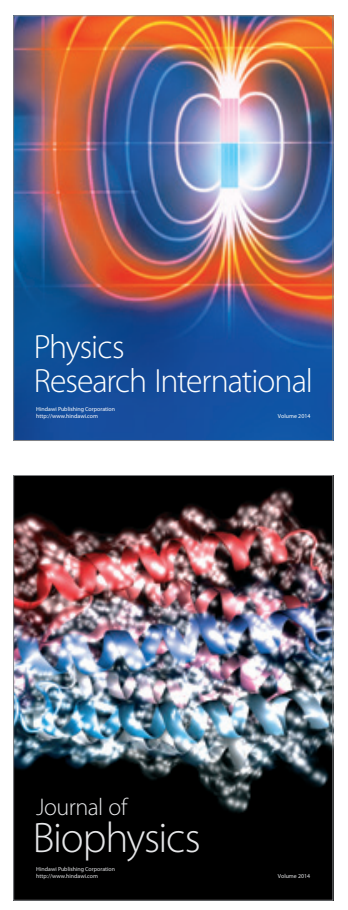
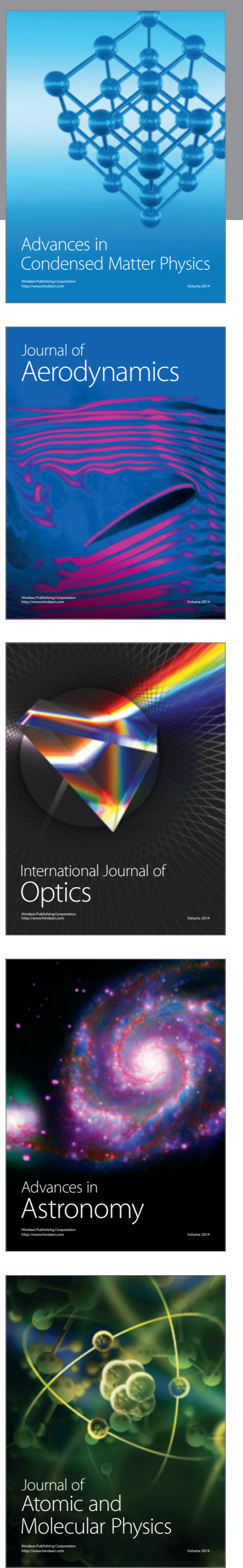